\title{
Critical frequency for vortex nucleation in Bose-Fermi mixtures in optical lattices
}

\author{
M. Guilleumas, M. Centelles, M. Barranco, R. Mayol, and M. Pi \\ Departament d'Estructura i Constituents de la Matèria, Facultat de Física, Universitat de Barcelona, Diagonal 647, 08028 Barcelona, \\ Spain
}

(Received 28 June 2005; published 3 November 2005)

\begin{abstract}
We investigate within mean-field theory the influence of a one-dimensional optical lattice and of trapped degenerate fermions on the critical rotational frequency for vortex line creation in a Bose-Einstein condensate. We consider laser intensities of the lattice such that quantum coherence across the condensate is ensured. We find a sizable decrease of the thermodynamic critical frequency for vortex nucleation with increasing applied laser strength and suggest suitable parameters for experimental observation. Since ${ }^{87} \mathrm{Rb}-{ }^{40} \mathrm{~K}$ mixtures may undergo collapse, we analyze the related question of how the optical lattice affects the mechanical stability of the system.
\end{abstract}

DOI: 10.1103/PhysRevA.72.053602

An essential feature of a rotating superfluid is the eventual appearance of quantized vortices [1]. For this reason, nucleation of vortices in dilute gases in rotating traps has been the subject of intensive experimental and theoretical research (see, e.g., Refs. [2-10] and references quoted therein).

Motivated by recent advances in the manipulation of rotating Bose-Einstein condensates (BEC) $[11,12]$ and of condensates loaded into optical lattices [13], and in anticipation of future experiments, we study a slowly rotating BEC in a one-dimensional (1D) optical lattice [14]. Rotating ultracold Bose gases in optical lattices are suitable systems to study quantum phenomena. In particular, the excitations, stability, and dynamics of a vortex line in the superfluid regime have been recently addressed [15]. Concurrently, the physics of degenerate Bose-Fermi mixtures in optical lattices is attracting conspicuous attention. Theoretical progress is being made in the basic understanding of the system's quantum phase diagram and the superfluid to Mott-insulator transition [16], and experimentalists have already prepared a degenerate mixture of $\mathrm{Rb}$ and $\mathrm{K}$ atoms in a tight optical lattice [17].

The aim of the present work is twofold. Firstly, to address the formation of vortices in a coherent array of Bose-Einstein condensates in a 1D optical lattice, and to determine the dependence of the thermodynamic critical angular velocity $\Omega_{c}$ for vortex formation [2,3] on the laser intensity. Secondly, to investigate the effect of trapped fermions on $\Omega_{c}$. Attractive Bose-Fermi mixtures may experience collapse and, thus, we also discuss the impact of the optical lattice on the mechanical stability of the mixture.

We consider a zero-temperature mixture made of a ${ }^{87} \mathrm{Rb}$ Bose condensate $(B)$ and a degenerate ${ }^{40} \mathrm{~K}$ Fermi gas $(F)$. They are confined by the axially symmetric external potentials of a harmonic magnetic trap and of a stationary periodic optical lattice modulated along the $z$ axis. The lattice is produced by a far detuned laser, which hinders the possibility of spontaneuous scattering and yields practically equal $\mathrm{Rb}$ and $\mathrm{K}$ optical potentials. The resulting potential for each kind of atom $q=B, F$ is
PACS number(s): 03.75.Lm, 03.75.Ss, 32.80.Pj

$$
V_{q}=\frac{1}{2} m_{q}\left(\omega_{q \perp}^{2} r^{2}+\omega_{q z}^{2} z^{2}\right)+\frac{V_{0}}{2} \cos (2 \pi z / d)
$$

where $m_{q}$ is the atomic mass. The radial and axial frequencies of the harmonic trap are taken from a recent experiment [13]: $\omega_{B \perp}=2 \pi \nu_{B \perp}$ with $\nu_{B \perp}=74 \mathrm{~Hz}$ and $\omega_{B z}=2 \pi \nu_{B z}$ with $\nu_{B z}=11 \mathrm{~Hz}$ for ${ }^{87} \mathrm{Rb}$, while those for ${ }^{40} \mathrm{~K}$ are a factor $\left(m_{B} / m_{F}\right)^{1 / 2} \simeq 1.47$ larger. The optical potential is determined by its period $d=2.7 \mu \mathrm{m}$ [13] and depth $V_{0}=s E_{R}$, where $E_{R}$ $=\hbar^{2} \pi^{2} / 2 m d^{2}=h(80 \mathrm{~Hz})$ is the recoil energy and $s$ is a dimensionless parameter.

For $3 \times 10^{5}$ atoms of ${ }^{87} \mathrm{Rb}$ confined in the harmonic trap, the condensate is cigar-shaped with a Thomas-Fermi length $L_{\mathrm{TF}}=84 \mu \mathrm{m}$, and a radius $R_{\mathrm{TF}}=6 \mu \mathrm{m}$. The superimposed optical potential splits the BEC over $L_{\mathrm{TF}} / d \sim 30$ wells. We shall be concerned with a range of laser intensities that ensures full coherence of the condensate across the whole system [15]. The criterion for the Mott transition [18] for the considered parameters leads to an estimate of a maximum lattice depth for the superfluid regime of $V_{0} \sim 150 E_{R} \sim h(12 \mathrm{kHz})$ [13], when quantum tunneling between consecutive wells is still sufficient to retain coherence.

Within our mean-field approach, neglecting $p$-wave interactions, the energy density functional that describes the boson-fermion mixture at zero temperature with a singly quantized vortex in the condensate along the $z$ axis has the form $[19,20]$

$$
\begin{aligned}
\mathcal{E}(\mathbf{r})= & \frac{\hbar^{2}}{2 m_{B}}(\nabla \Psi)^{2}+\frac{\hbar^{2}}{2 m_{B}} \frac{1}{r^{2}} n_{B}+V_{B} n_{B}+\frac{1}{2} g_{B B} n_{B}^{2}+g_{B F} n_{F} n_{B} \\
& +\frac{\hbar^{2}}{2 m_{F}} \tau_{F}+V_{F} n_{F}
\end{aligned}
$$

where $n_{B}=|\Psi|^{2}$ is the condensate density, $n_{F}$ is the fermion density, and $\tau_{F}$ is the fermion kinetic energy density written as a function of $n_{F}$ and its gradients [Thomas-FermiWeizsäcker (TFW) approximation]. The boson-boson and boson-fermion coupling constants $g_{B B}$ and $g_{B F}$ are written in terms of the $s$-wave scattering lengths $a_{B}$ and $a_{B F}$ as $g_{B B}$ 

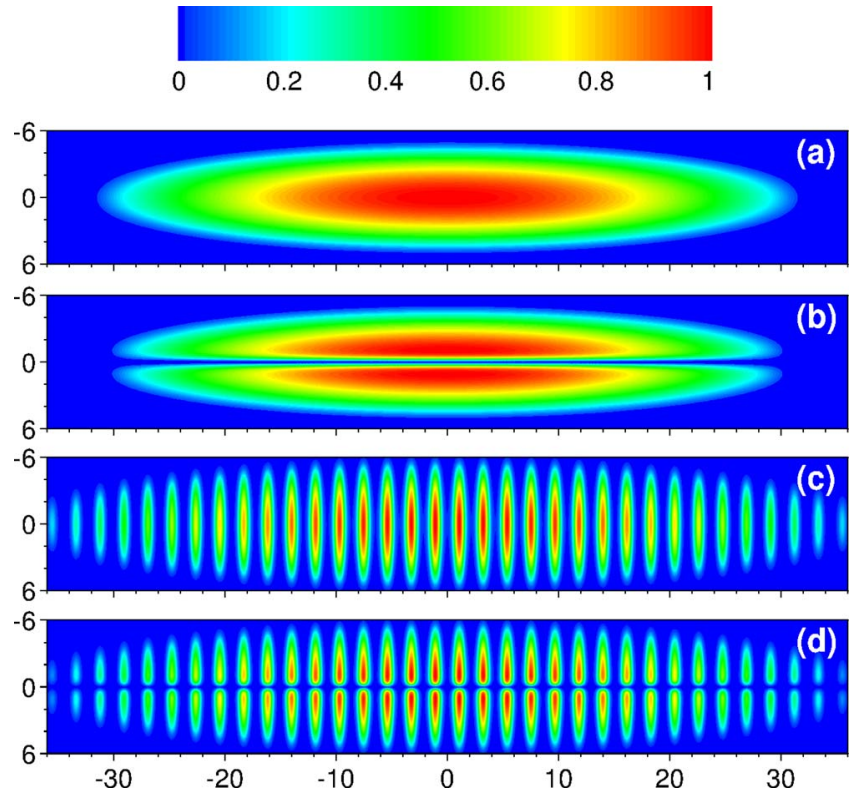

FIG. 1. (Color online) Contour plots of the ${ }^{87} \mathrm{Rb}$ condensate density in the $x z$ plane. (a) Condensate in the pure harmonic trap. (b) Same as (a) with a quantized vortex along the $z$ axis. (c) Same as (a) under an optical lattice with $V_{0} / h=2.5 \mathrm{kHz}$. (d) Same as (c) with a quantized vortex along the $z$ axis. Distances are in units of the oscillator length $a_{B \perp}=\sqrt{\hbar /\left(m_{B} \omega_{B \perp}\right)}=1.25 \mu \mathrm{m}$. The density scale from 0 to 1 at the top of the figure is normalized to the highest density in each panel [these are 229 in (a), 212 in (b), 394 in (c), and $376 a_{B \perp}^{-3}$ in $\left.(\mathrm{d})\right]$.

$=4 \pi a_{B} \hbar^{2} / m_{B}$ and $g_{B F}=4 \pi a_{B F} \hbar^{2} / m_{B F}$, respectively, with $m_{B F} \equiv 2 m_{B} m_{F} /\left(m_{B}+m_{F}\right)$.

The variation of $\mathcal{E}$ with respect to $n_{B}$ and $n_{F}$, under the constraint of given number of bosons $N_{B}$ and fermions $N_{F}$, yields two coupled Euler-Lagrange equations: a GrossPitaevskii equation for bosons with a term describing the boson-fermion interaction, and a TFW equation for fermions $[19,20]$, which is increasingly valid the larger $N_{F}$ is. Starting from randomly sampled densities, we solve these equations as indicated in Ref. [21]. We use the adapted version of the virial theorem [20] to check the numerical convergence.

We first consider a pure BEC, with $N_{B}=3 \times 10^{5}$ atoms of ${ }^{87} \mathrm{Rb}$ as in the experiment of Ref. [13]. For the boson-boson scattering length we use the value $a_{B}=98.98 a_{0} \quad\left(1 a_{0}\right.$ $=0.529 \AA$ ) $[22]$. In Fig. 1 we show contour plots of the condensate density in the $x z$ plane, $n_{B}(x, 0, z)=|\Psi(x, 0, z)|^{2}$, for different situations. In (a) the condensate is confined only by the harmonic trap. In (b) the cigar-shaped condensate of (a) hosts a quantized vortex line along the $z$ axis. In (c) a 1D optical lattice with $V_{0} / h=2.5 \mathrm{kHz}$ splits the cigar-shaped condensate (a) into an array of multiple disklike coherent condensates. As shown in Ref. [23], certain aspects of the macroscopic properties and low-energy dynamics of a magnetically trapped BEC in a tight optical lattice can be understood in terms of a renormalized interaction coupling constant $g_{B B}^{*}>g_{B B}$ and of an effective mass $m_{B}^{*}>m_{B}$ along the direction of the periodic optical potential. The increase of the radial size of the sample that one observes in panel (c) with respect to panel (a) is due to the increased repulsive effect of

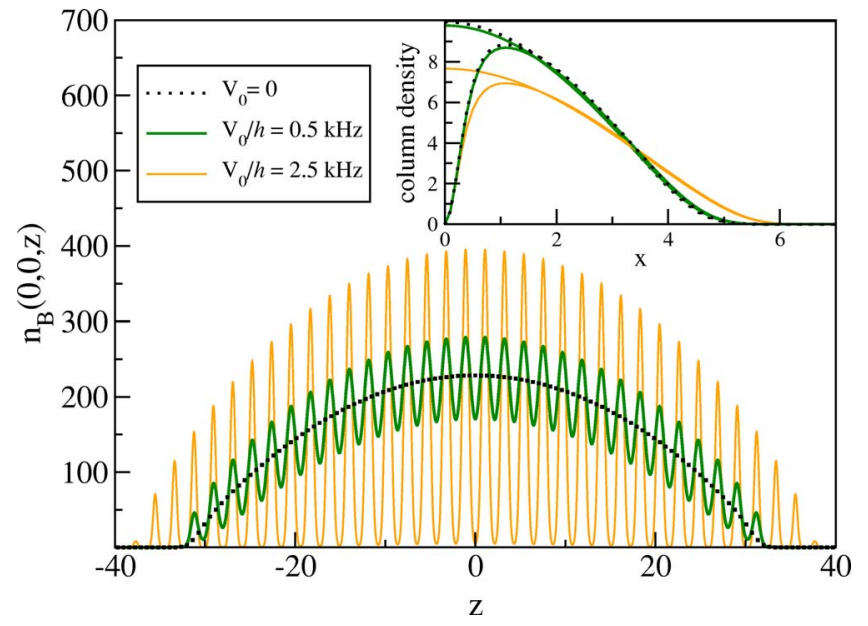

FIG. 2. (Color online) Density profile of the condensate along the $z$ axis $n_{B}(0,0, z)=|\Psi(0,0, z)|^{2}$, in units of $a_{B \perp}^{-3}$, for different laser intensities. The coordinate $z$ is in units of $a_{B \perp}$. Inset: column density $n_{B}(x)$, in units of $10^{3} \times a_{B \perp}^{-2}$, for the same $V_{0}$ values. The column density of the condensate when it hosts a vortex line is also plotted. The coordinate $x$ is in units of $a_{B \perp}$.

the boson-boson interaction when the system feels the optical lattice. It originates from the fact that the optical confinement produces a local compression of the gas inside each well $[18,23]$. The axial size of the condensate also increases appreciably due to the combined effect of the repulsive interactions and the redistribution of atoms inside the potential wells. In panel (d) of Fig. 1 the optical lattice is superimposed to the condensate with the vortex state (b). In a bulk superfluid, the size of the vortex core is of the order of the healing length $\xi=\left[8 \pi n_{B} a\right]^{-1 / 2}$, where $n_{B}$ is the bulk density. This expression holds for inhomogeneous superfluids taking for $n_{B}$ the local boson density in the absence of vortices [3]. Thus, the vortex core size is larger for the outer sites of the split condensate, where the density is smaller.

Figure 2 shows the evolution of the density profile of the condensate along the $z$ axis, $n_{B}(0,0, z)=|\Psi(0,0, z)|^{2}$, with the laser intensity. In the presence of a shallow 1D optical lattice, the condensate profile starts developing small oscillations around the profile at $V_{0}=0$ which follow the periodic optical potential, but its spatial extension is nearly not affected. When the laser intensity increases the system nearly splits into separate condensates. The effect of the local compression inside each potential well of the lattice, as well as the increase of the axial size of the system, can be clearly seen in Fig. 2. In the inset we plot the column density $n_{B}(x)=\int d z n_{B}(x, 0, z)$ for the condensate in the harmonic trap alone and when the optical lattice is added. We also display $n_{B}(x)$ for the condensate hosting a vortex line, in this case the quantized circulation around the vortex line pushes the atoms away from it.

The thermodynamic critical angular velocity $\Omega_{c}$ for nucleating a singly quantized vortex is obtained by subtracting from the vortex state energy $E_{1}$ in the rotating frame the ground-state energy $E_{0}$, i.e., $\Omega_{c}=\left(E_{1} / N_{B}-E_{0} / N_{B}\right) / \hbar[2,3]$, and it provides a lower bound to the critical angular velocity [3]. In Fig. 3 we plot $\Omega_{c}$ as a function of the laser intensity 


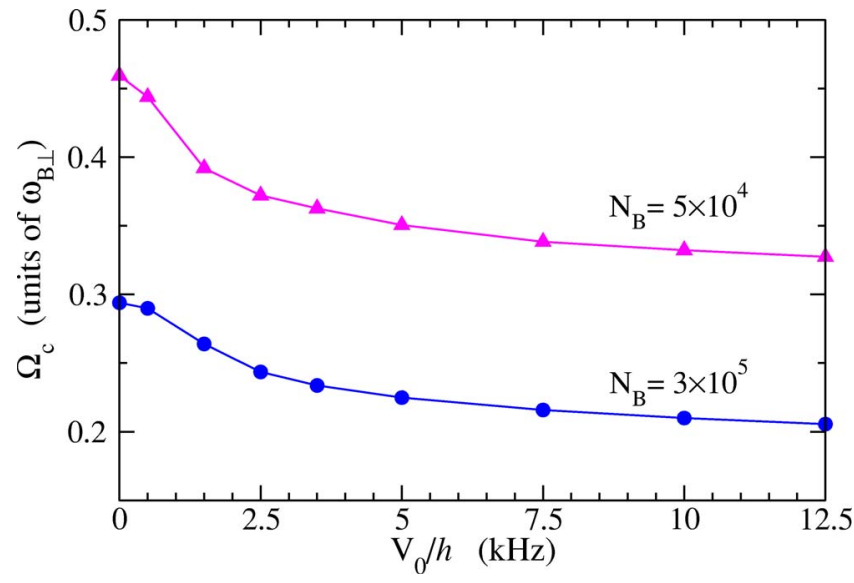

FIG. 3. (Color online) Critical angular velocity $\Omega_{c}$ for nucleation of a singly quantized vortex line as a function of the laser intensity $V_{0}$.

for $N_{B}=3 \times 10^{5}$ and for $N_{B}=5 \times 10^{4}$. For a fixed number of condensate atoms, $\Omega_{c}$ decreases when $V_{0}$ increases, in agreement with the fact that the radial size of the system becomes larger in the presence of the optical lattice and the associated reduction of atoms along the symmetry axis (see the decrease of the column density $n_{B}$ at the origin in Fig. 2 with increasing $V_{0}$ ). In turn, the increase of the inertia of the condensate along the direction of the laser beam due to the larger effective mass $m_{B}^{*}[23]$ also contributes to the reduction of the critical angular velocity.

Assuming that the relative effect remains of the same order in experiment, Fig. 3 predicts a sizable $24 \%$ reduction of $\Omega_{c}$ already with a laser strength $V_{0} / h=5 \mathrm{kHz}$ for both of the $N_{B}$ values. The effect is enhanced the shallower the magnetic trap is. A less elongated magnetic trap would favor the appearence of a vortex at a slower rotation. For example, the values of $\Omega_{c}\left(V_{0}=0\right)$ shown in Fig. 3 would be decreased by a 0.6 factor in a spherical trap with $\nu_{B z}=\nu_{B \perp}=74 \mathrm{~Hz}$. The relative reduction of $\Omega_{c}$ caused by $V_{0} / h=5 \mathrm{kHz}$ with respect to $V_{0}=0$ would still be of $20-23 \%$.

Figure 3 suggests an experiment to test the dependence of the critical frequency for vortex nucleation on the intensity of the optical lattice, since it shows that, for a rotating condensate with a given $N_{B}$, a vortex line should nucleate at angular frequencies lower than $\Omega_{c}\left(V_{0}=0\right)$ if a corotating 1D optical lattice is superimposed, whereas otherwise it would not. As the thermodynamic $\Omega_{c}$ underestimates the actual critical frequency for vortex creation, dynamical calculations are needed for a precise quantitative estimate of this effect.

With the purpose of assessing the effects of a trapped fermion cloud on the rotating condensate in an optical lattice, we consider next a ${ }^{87} \mathrm{Rb}-{ }^{40} \mathrm{~K}$ mixture. It is characterized by a large attractive Bose-Fermi interaction that assists the sympathetic cooling of the fermionic species down to the degenerate regime. There are various values of the interspecies scattering length $a_{B F}$ published, depending on the type of experiment and techniques used [24-27]. A recent measurement of $a_{B F}$ at JILA [27] has established a value $a_{B F}=-250 \pm 30 a_{0}$, which we adopt here because of its reduced experimental uncertainties. Vortex states in ${ }^{87} \mathrm{Rb}-{ }^{40} \mathrm{~K}$

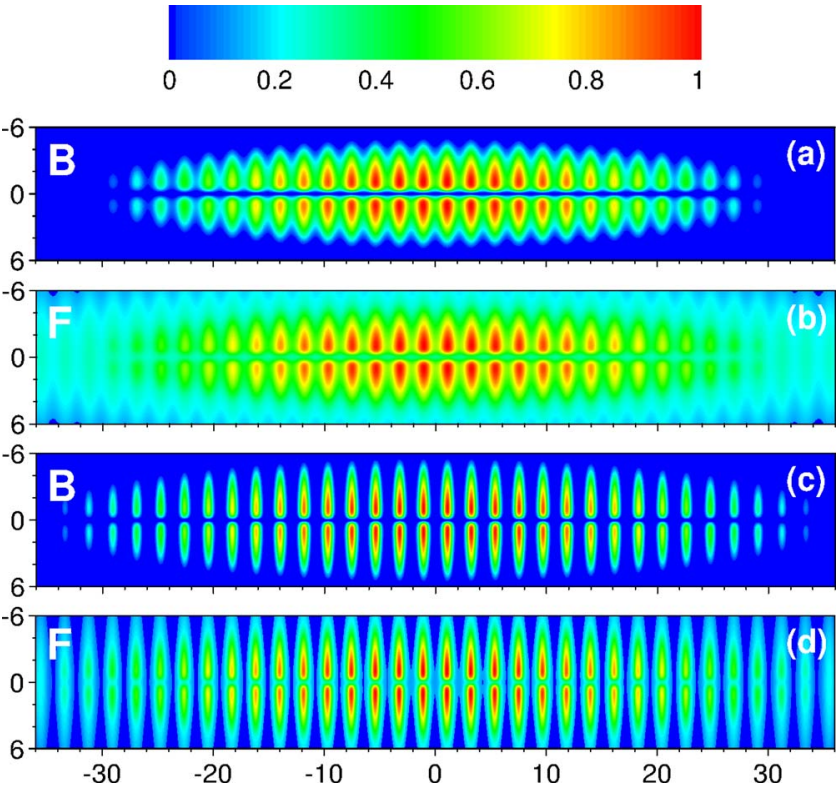

FIG. 4. (Color online) Contour plots of the condensate (B) and fermionic (F) densities in the $x z$ plane for the ${ }^{87} \mathrm{Rb}-{ }^{40} \mathrm{~K}$ mixture, in the combined harmonic and optical lattice trap. The condensate hosts a quantized vortex. In (a) and (b) $V_{0} / h=0.5 \mathrm{kHz}$, whereas in (c) and (d) $V_{0} / h=2.5 \mathrm{kHz}$. Distances are in units of $a_{B \perp}$. The density scale from 0 to 1 at the top of the figure is normalized to the highest density in each panel [these are 312 in (a), 25 in (b), 457 in (c), and $37 a_{B \perp}^{-3}$ in (d)].

mixtures confined by a harmonic potential have been recently addressed [20] (although $a_{B F}=-395 a_{0}$ [26] was employed). When a $1 \mathrm{D}$ optical lattice is switched on, the atomic species experience the trapping potential (1).

We have carried out calculations for a mixture with $N_{B}$ $=3 \times 10^{5}$ and $N_{F}=1.5 \times 10^{5}$ atoms, and the same parameters as in Fig. 1. We assume that the Fermi component is in the normal — nonsuperfluid — but quantum degenerate phase, and consider that it is in a stationary state. This situation could be achieved experimentally by waiting long enough after the generation of the vortex in the condensate to let the drag force between bosons and fermions to dissipate. We present in Fig. 4 contour plots of the boson $n_{B}(x, 0, z)$ and fermion $n_{F}(x, 0, z)$ densities when the condensate hosts a vortex line, for two laser strengths: $V_{0} / h=0.5$ [Figs. 4(a) and 4(b)] and $2.5 \mathrm{kHz}$ [Figs. 4(c) and 4(d)]. The condensate and the fermionic cloud are modulated by the regular pattern of the optical lattice. The large mutual attraction between fermions and bosons makes the effective interaction between bosons less repulsive than in the pure BEC. Furthermore, the boson atoms induce an effective attraction between fermions counteracting the Fermi pressure. As a result, the density of both species increases in the overlapping region, as if they were more strongly confined by the external potentials, and the condensate becomes more compact in space [compare Figs. 4(c) and 1(d)]. Still, the effect of the Pauli exclusion principle is notorious in Fig. 4, where the ${ }^{40} \mathrm{~K}$ cloud is seen to extend to larger distances than the ${ }^{87} \mathrm{Rb}$ atoms, both axially and radially.

The presence of a vortex along the $z$ axis in the conden- 
sate component is apparent in panels (a) and (c) of Fig. 4 as the boson density vanishes on the vortex line. It is interesting to note that the large attractive $a_{B F}$ leads to a visible depletion of the fermionic density on the $z$ axis as well, reminiscent of the bosonic vortex core. The effect should be directly observable experimentally. It is more evident in Fig. 4(d), where bosons and fermions are more tightly confined by the optical lattice.

In the present mixture we find that the presence of the quantum degenerate fermionic cloud does not change much the value of $\Omega_{c}$ compared to the pure BEC, nor its pattern against the optical lattice strength. Indeed, for all the $V_{0}$ values considered here the critical frequency for vortex appearence is raised by $\sim 10 \%$, due to the enhancement of the condensate density at the core caused by the fermion atoms. The impact of the fermion cloud on $\Omega_{c}$ is magnified in a deeper magnetic trap. For instance, if the same mixture was set in a trap with $\nu_{B z}=95 \mathrm{~Hz}$ and $\nu_{B \perp}=640 \mathrm{~Hz}$, that preserves the aspect ratio $\nu_{B z} / \nu_{B \perp}=0.15$ used in Fig. 4, the critical frequency $\Omega_{c}\left(V_{0}=0\right)$ would increase by $40 \%$ with respect to the $N_{F}=0$ case. In this squeezing magnetic trap, however, the effect of $V_{0}$ on $\Omega_{c}$ is negligible $(<2 \%)$.

A peculiar feature of the ${ }^{87} \mathrm{Rb}-{ }^{40} \mathrm{~K}$ system, stemming from the interplay of the moderately repulsive $a_{B}$ and the strongly attractive $a_{B F}$, is the existence of a mechanical stability limit beyond which the system cannot sustain more fermions, for at high densities the strong interspecies attraction may overcome the Pauli pressure and drive the ultracold gas mixture into collapse. The phenomenon has been observed experimentally [25]. Thus, we next address the stability of the ${ }^{87} \mathrm{Rb}-{ }^{40} \mathrm{~K}$ system subject to an optical lattice. In fact, collapse is currently the focus of experimental attention because of the implications the instability has for constraining $a_{B F}[25-27]$, and also because there are prospects that a radially squeezed ${ }^{87} \mathrm{Rb}-{ }^{40} \mathrm{~K}$ mixture at a density close to collapse must be able to form stable bright soliton trains [28].

Keeping $N_{B}$ fixed at $3 \times 10^{5}$ atoms as in the previous dis- cussions, with $a_{B F}=-250 a_{0}$ we find that the boson-fermion mixture confined in the magnetic trap of Fig. 4 would be stable up to virtually arbitrary fermion numbers, consistent with the findings of Ref. [19]. Increasing $\omega_{B \perp}$ prompts the occurrence of collapse. In a trap with $\nu_{B z}=95 \mathrm{~Hz}$ and $\nu_{B \perp}$ $=640 \mathrm{~Hz}$, the $3 \times 10^{5}$ rubidium atoms are able to retain up to a maximum of $N_{F}^{\mathrm{max}}=2.6 \times 10^{5}$ fermions, when the system collapses. (The effect on $\Omega_{c}$ of $N_{F}=1.5 \times 10^{5}$ and $V_{0}$ for this trap has been discussed above.) Now, a decrease of $N_{F}^{\max }$ can be seen when a 1D optical lattice is added, caused by the effective local compression of the atoms inside the optical trap. Indeed, superimposing a $1 \mathrm{D}$ optical lattice with $d$ $=2.7 \mu \mathrm{m}$ and $V_{0} / h=5 \mathrm{kHz}$ to this harmonic trap, the ${ }^{87} \mathrm{Rb}-$ ${ }^{40} \mathrm{~K}$ mixture with $N_{B}=3 \times 10^{5}$ can sustain up to $N_{F}^{\max }$ $=7.5 \times 10^{4}$ fermions only, i.e., less than $30 \%$ of the $V_{0}=0$ value. The effect appears to be accessible to verification under present experimental conditions. It opens the possibility to study the collapse of a mixture for trapped fermion and boson numbers considerably smaller than in a pure harmonic trap.

In conclusion, we have determined the thermodynamic critical frequency for nucleating a quantized vortex in an array of coherent condensates within the Gross-Pitaevskii theory. We have studied a Bose-Fermi mixture trapped in a $1 \mathrm{D}$ optical lattice to examine the effect on the value of $\Omega_{c}$ of a fermionic cloud in the quantum degenerate phase. ${ }^{87} \mathrm{Rb}$ ${ }^{40} \mathrm{~K}$ mixtures may collapse and, in this regard, it has been shown that a 1D optical lattice can be an efficient degree of freedom to tune the onset of instability. Our analysis of the relative variation of $\Omega_{c}$ calculated by thermodynamic arguments is intended to provide useful information for future experiments. A quantitative determination of $\Omega_{c}$ requires dynamical calculations. Work in this line is being undertaken.

We thank Dr. K. Bongs for valuable discussions. This work has been performed under Grants No. BFM2002-01868 from DGI (Spain) and FEDER, and No. 2001SGR-00064 from Generalitat de Catalunya. M.G. thanks the "Ramón y Cajal” Program (Spain) for financial support.
[1] R. J. Donnelly, Quantized Vortices in Helium II (Cambridge University Press, Cambridge, 1991).

[2] F. Dalfovo and S. Stringari, Phys. Rev. A 53, 2477 (1996).

[3] A. L. Fetter and A. A. Svidzinsky, J. Phys.: Condens. Matter 13, R135 (2001).

[4] J. J. García-Ripoll and V. M. Pérez-García, Phys. Rev. A 60, 4864 (1999).

[5] Ph. W. Courteille, V. S. Bagnato, and V. I. Yukalov, Laser Phys. 11, 659 (2001).

[6] C. J. Pethick and H. Smith, Bose-Einstein Condensation in Dilute Gases (Cambridge University Press, Cambridge, 2002).

[7] L. Pitaevskii and S. Stringari, Bose-Einstein Condensation (Clarendon, Oxford, 2003).

[8] M. R. Matthews, B. P. Anderson, P. C. Haljan, D. S. Hall, C. E. Wieman, and E. A. Cornell, Phys. Rev. Lett. 83, 2498 (1999).

[9] K. W. Madison, F. Chevy, W. Wohlleben, and J. Dalibard, Phys. Rev. Lett. 84, 806 (2000).
[10] J. R. Abo-Shaeer, C. Raman, J. M. Vogels, and W. Ketterle, Science 292, 476 (2001).

[11] P. Rosenbusch, V. Bretin, and J. Dalibard, Phys. Rev. Lett. 89, 200403 (2002).

[12] V. Bretin, S. Stock, Y. Seurin, and J. Dalibard, Phys. Rev. Lett. 92, 050403 (2004).

[13] Z. Hadzibabic, S. Stock, B. Battelier, V. Bretin, and J. Dalibard, Phys. Rev. Lett. 93, 180403 (2004).

[14] Fast-rotating condensates hosting a large number of vortices are also currently investigated, see, e.g., M. Tsubota, K. Kasamatsu, and M. Ueda, Phys. Rev. A 65, 023603 (2002); P. Engels, I. Coddington, P. C. Haljan, V. Schweikhard, and E. A. Cornell, Phys. Rev. Lett. 90, 170405 (2003); H. Zhai, Qi Zhou, R. Lü, and L. Chang, Phys. Rev. A 69, 063609 (2004); A. L. Fetter, B. Jackson, and S. Stringari, ibid. 71, 013605 (2005).

[15] J.-P. Martikainen and H. T. C. Stoof, Phys. Rev. Lett. 91, 
240403 (2003); Phys. Rev. A 69, 053617 (2004); P. G. Kevrekidis, R. Carretero-González, G. Theocharis, D. J. Frantzeskakis, and B. A. Malomed, J. Phys. B 36, 3467 (2003); A. B. Bhattacherjee, O. Morsch, and E. Arimondo, ibid. 37, 2355 (2004); J. W. Reijnders and R. A. Duine, Phys. Rev. Lett. 93, 060401 (2004).

[16] M. Lewenstein, L. Santos, M. A. Baranov, and H. Fehrmann, Phys. Rev. Lett. 92, 050401 (2004); M. Cramer, J. Eisert, and F. Illuminati, ibid. 93, 190405 (2004).

[17] G. Modugno, F. Ferlaino, R. Heidemann, G. Roati, and M. Inguscio, Phys. Rev. A 68, 011601(R) (2003).

[18] W. Zwerger, J. Opt. B: Quantum Semiclassical Opt. 5, S9 (2003).

[19] R. Roth, Phys. Rev. A 66, 013614 (2002).

[20] D. M. Jezek, M. Barranco, M. Guilleumas, R. Mayol, and M. Pi, Phys. Rev. A 70, 043630 (2004).

[21] M. Barranco, M. Guilleumas, E. S. Hernández, R. Mayol, M.
Pi, and L. Szybisz, Phys. Rev. B 68, 024515 (2003).

[22] E. G. M. van Kempen, S. J. J. M. F. Kokkelmans, D. J. Heinzen, and B. J. Verhaar, Phys. Rev. Lett. 88, 093201 (2002).

[23] M. Krämer, L. Pitaevskii, and S. Stringari, Phys. Rev. Lett. 88, 180404 (2002).

[24] G. Ferrari, M. Inguscio, W. Jastrzebski, G. Modugno, G. Roati, and A. Simoni, Phys. Rev. Lett. 89, 053202 (2002).

[25] G. Modugno, G. Roati, F. Riboli, F. Ferlaino, R. J. Brecha, and M. Inguscio, Science 297, 2240 (2002).

[26] M. Modugno, F. Ferlaino, F. Riboli, G. Roati, G. Modugno, and M. Inguscio, Phys. Rev. A 68, 043626 (2003).

[27] J. Goldwin, S. Inouye, M. L. Olsen, B. Newman, B. D. DePaola, and D. S. Jin, Phys. Rev. A 70, 021601(R) (2004).

[28] T. Karpiuk, M. Brewczyk, S. Ospelkaus-Schwarzer, K. Bongs, M. Gajda, and K. Rzążewski, Phys. Rev. Lett. 93, 100401 (2004). 